\title{
Peculiaridades da circulação mesentérica em recém-nascidos e suas implicações em doenças gastrintestinais do período neonatal
}

\author{
Mesenteric circulation peculiarities in infant newborns and its implications in gastrointestinal \\ illnesses of the neonatal period
}

Chang Yin Chia ${ }^{1}$, Mário Cícero Falcão

\section{RESUMO}

Objetivo: Descrever peculiaridades da circulação mesentérica neonatal e caracterizar fatores de suscetibilidade ao desenvolvimento de doenças gastrintestinais e alterações do fluxo sanguíneo da artéria mesentérica superior por meio da dopplerfluxometria.

Fontes de dados: Livros-textos e publicações indexadas no Medline e SciELO nos últimos 20 anos, utilizando-se as palavras chaves: "mesenteric artery", "superior mesenteric artery", "newborn intestinal circulation", "necrotizing enterocolitis", "doppler flow velocimetry".

Síntese dos dados: Alterações do fluxo sanguíneo mesentérico são um dos fatores predisponentes da enterocolite necrosante, doença neonatal de alta morbimortalidade que acomete principalmente prematuros. A circulação mesentérica é peculiar no período neonatal tanto em relação ao seu estado basal, quanto à sua resposta frente a estímulos fisiológicos. Variações da irrigação mesentérica podem ser inerentes à própria fase de desenvolvimento vascular intestinal do recém-nascido pré-termo, com possíveis agravos de fatores perinatais como: insuficiência placentária, asfixia, infecção, cateterismo umbilical, drogas (indometacina e cafeína), fototerapia, alimentação artificial e progressão rápida da dieta. A dopplerfluxometria permite o estudo da irrigação de órgãos-alvo e pode quantificar o fluxo sanguíneo, a resistência vascular e predizer situações de risco para doenças do trato gastrintestinal no período neonatal.

Conclusões: O recém-nascido apresenta peculiaridades de irrigação sanguínea gastrintestinal. A dopplerfluxometria da artéria mesentérica superior é um método não

Instituição: Departamento de Pediatria da Faculdade de Medicina da Universidade de São Paulo (FMUSP), São Paulo, SP, Brasil

'Mestre em Ciências da Saúde pelo Departamento de Pediatria da FMUSP; Médica do Serviço de Neonatologia do Hospital e Maternidade São Luiz, São Paulo, SP, Brasil

${ }^{2}$ Doutor em Pediatria pelo Departamento de Pediatria da FMUSP; Médico Encarregado da Unidade de Cuidados Intensivos Neonatal do Instituto da Criança do Hospital das Clínicas da FMUSP; Docente da Universidade Anhembi-Morumbi, Laureate Universities, São Paulo, SP, Brasil invasivo que determina as condições circulatórias no território intestinal.

Palavras-chave: circulação esplâncnica; artéria mesentérica superior; enterocolite necrosante; ultrassom; recémnascido.

\section{ABSTRACT}

Objective: To describe peculiarities of the neonatal mesenteric circulation and to characterize the susceptibility factors to the development of gastrointestinal illnesses and alterations of the superior mesenteric artery flow by the Doppler ultrasound method.

Data sources: Textbooks and publications listed in Medline and SciELO from the last 20 years were searched using the key-words: "mesenteric artery", "intestinal superior mesenteric artery", "newborn circulation", "necrotizing enterocolitis", and "Doppler flow velocimetry".

Data synthesis: Alterations in the mesenteric flow are predisposing factors for necrotizing enterocolitis, which is a neonatal illness with high morbidity and mortality that occurs mainly in premature infants. The mesenteric circulation is peculiar at the neonatal period regarding its baseline status as well as its response from physiological stimulation. Mesenteric irrigation varies according to the proper phase of intestinal vascular development. Mesenteric irrigation can be damaged by perinatal factors, such as placental insufficiency, asphyxia, infection, umbilical catheters, drugs (indomethacin and caffeine), phototherapy, artificial feeding and fast diet progression. Doppler flow velocimetry allows

Endereço para correspondência:

Mário Cícero Falcão

Rua Vieira de Moraes, 45, apto. 51

CEP 04617-010 - São Paulo/SP

E-mail: profmariofalcao@yahoo.com.br

Recebido em: 4/5/08

Aceito em: 14/9/08 
the study of gut irrigations and may quantify blood flow and vascular resistance. The exam can predict risk situations for gastrointestinal illnesses during the neonatal period.

Conclusions: The newborn infant presents peculiarities on gastrointestinal blood circulation. Superior mesenteric artery Doppler ultrasound provides non-invasive measures of the intestinal circulatory conditions.

Key-words: splanchnic circulation; mesenteric artery, superior; enterocolitis, necrotizing; ultrasonics; infant, newborn.

\section{Introdução}

A regulação do fluxo sanguíneo no território mesentérico ocorre em dois pontos, nas arteríolas e no esfíncter précapilar, e em dois níveis de controle, intrínseco e extrínseco. O controle intrínseco é dependente da pressão arterial de oxigênio; a vasodilatação ocorre como resposta à oclusão do fluxo local (hiperemia reacional) ou à alimentação enteral (hiperemia funcional), envolvendo mudanças no tônus vascular tanto no nível arteriolar como pré-capilar. A regulação extrínseca é mediada pelo estímulo simpático dos nervos esplâncnicos e sofre influência de fatores circulantes endógenos e exógenos, como hormônios, histamina e prostaglandinas e componentes da dieta enteral. Por outro lado, há situações em que os dois mecanismos interagem, fenômeno conhecido como escape autorregulatório, que ocorre quando o fluxo sanguíneo mesentérico é comprometido pelo estímulo de nervos periarteriolares e, em poucos minutos, é restaurado por regulação intrínseca ${ }^{(1)}$.

Assim, o objetivo do presente estudo é descrever peculiaridades da circulação mesentérica neonatal e caracterizar os fatores de suscetibilidade no desenvolvimento de doenças gastrintestinais e as alterações do fluxo sanguíneo da artéria mesentérica superior por meio da dopplerfluxometria. Para tal, foram consultadas as seguintes fontes de dados: livros textos e publicações indexadas no Medline e SciELO por meio de pesquisa utilizando-se as palavras-chave: "mesenteric artery", "superior mesenteric artery", "newborn intestinal circulation", "necrotizing enterocolitis", "doppler flow velocimetry”, publicados nos últimos 20 anos. Para a discussão, os textos selecionados foram agrupados em quatro subitens: peculiaridades da circulação mesentérica, mecanismos reguladores da circulação intestinal, resposta vascular da circulação intestinal e características da circulação intestinal na patogênese da enterocolite necrosante.

\section{Peculiaridades da circulação mesentérica no recém-nascido}

O fluxo sanguíneo intestinal no recém-nascido é regulado por mecanismos intrínsecos e extrínsecos, assim como por substâncias vasoativas circulantes. Alterações de velocidade e características desse fluxo podem ser relacionadas a fatores de morbidade perinatal ${ }^{(2)}$.

A teoria da isquemia intestinal relacionada à redistribuição do fluxo sanguíneo como patogênese da enterocolite necrosante foi inicialmente proposta por Lloyd, em $1969^{(3)}$. Essa redistribuição teria origem na resposta neurogênica frente à redução do débito cardíaco, levando ao comprometimento dos órgãos esplâncnicos, com necrose intestinal e perfuração.

A enterocolite necrosante é uma doença da infância precoce. Sendo assim, pode-se supor que condições fisiológicas intestinais são peculiares do recém-nascido, assim como fatores circulatórios fisiológicos do intestino do recém-nascido são dependentes da idade. Outro fator que interfere na perfusão intestinal é a policitemia, que leva à hiperviscosidade sanguínea, gerando redução da velocidade do fluxo sanguíneo local, podendo reduzir a oxigenação intestinal em até $50 \%{ }^{(4)}$.

Uma das primeiras observações a respeito das características fisiológicas circulatórias do intestino do recém-nascido foi feita por Crissinger et al, em 1988 ${ }^{(5)}$. Esses autores demonstraram, em modelos animais, que o intestino do recém-nascido apresenta maior consumo de oxigênio. Esse aumento é necessário para atender à demanda dos processos digestivos do intestino, assim como para o seu crescimento, participando do preparo para que o intestino se torne a única via de aquisição de nutrientes. Histologicamente, observase, ainda, um aumento de capilares perfusionais, ampliando a superfície de difusão do oxigênio aos intestinos. Essas características tornam o intestino do recém-nascido mais suscetível à hipóxia.

Ademais, o intestino do recém-nascido não apresenta uma resposta adequada à hipotensão sistêmica, não sendo capaz de reduzir sua resistência vascular local e relaxar os esfíncteres pré-capilares de maneira satisfatória, necessitando recorrer ao mecanismo de escape autorregulatório, mediado por inervação simpática, para aumentar o fluxo sanguíneo local nessas condições.

As células endoteliais são responsáveis pela produção de agentes vasoativos que atuam no tônus da musculatura lisa vascular local. Um desses agentes é o óxido nítrico, responsável pelo relaxamento do tônus vascular. Assim, processos inflamatórios locais ou sistêmicos com danos na vasculatura 
local podem levar à redução do fluxo sanguíneo e comprometimento da resposta vascular fisiológica, agravando o processo da lesão intestinal ${ }^{(6)}$.

Em condições habituais, após um período de privação de oxigênio, a vasculatura regional responde de maneira danosa à reperfusão, com aumento de produção de radicais livres, o que provoca lesão extensa do tecido acometido. Esse processo ocorre por meio de dois mecanismos; por um lado, há a ativação de neutrófilos que se aderem ao endotélio vascular e, por outro, as xantinas oxidases ligadas ao tecido geram radicais livres a partir da degradação enzimática da hipoxantina. No recém-nascido, o intestino tem uma capacidade limitada de gerar radicais livres pela menor atividade da xantina oxidase e menor quantidade de neutrófilos aderidos ao endotélio. Porém, esse mecanismo de lesão pode estar exacerbado em recém-nascidos expostos à dieta enteral formulada, relacionando-se ao conteúdo dietético de lipídios ${ }^{(7)}$.

O fator ativador de plaquetas é um fosfolipídio produzido por diversos tecidos, inclusive pelo parênquima intestinal e endotélio vascular, e induzido por hipóxia, possuindo uma atividade vasoconstritora potente ${ }^{(8)}$. A sua ação na microvasculatura intestinal pode ser direta ou mediada pela liberação de leucotrieno $\mathrm{C} 4$, também potente vasoconstritor ${ }^{(9)}$.

Logo após o nascimento, o fluxo mesentérico se reduz pela transição da circulação fetal para a neonatal, que persiste, em geral, até três dias, período durante o qual o ducto arterioso permanece patente. Comprometimento do estado hemodinâmico, como choque ou hipotensão, gera redistribuição do fluxo sistêmico, priorizando-se a irrigação de territórios mais nobres, com vasoconstrição mesentérica. $\mathrm{O}$ uso de drogas vasoativas nessas situações pode tanto melhorar como reduzir a perfusão mesentérica, seja por ação direta local da droga ou por variação da condição hemodinâmica ${ }^{(10)}$.

A administração da indometacina para tratamento da persistência do ducto arterial leva à vasoconstricção mesentérica, comprometendo a perfusão intestinal. A cafeína, utilizada para tratamento da apneia da prematuridade, leva à redução do fluxo sanguíneo cerebral e intestinal, sem alterar as condições hemodinâmicas ${ }^{(11)}$.

Quadros infecciosos locais ou sistêmicos geram citoquinas inflamatórias que comprometem o fluxo sanguíneo mesentérico, sendo a mais conhecida delas a endotelina-1, um potente vasoconstritor, porém, em sepse neonatal precoce, pode ocorrer resposta inflamatória sistêmica semelhante à do adulto, com hiperemia do leito esplâncnico ${ }^{(12)}$.

A irrigação mesentérica pode sofrer também alterações conforme o tipo de fototerapia utilizada para tratamento de icterícia. A fototerapia convencional bloqueia a hiperemia funcional em resposta à dieta ${ }^{(13)}$.

O cateterismo de vasos umbilicais para manutenção de acesso vascular e monitorização hemodinâmica tem sido relacionado ao aumento de risco para condições de morbidade do sistema intestinal pela redução do fluxo sanguíneo efetivo $^{(11)}$.

Prematuros apresentam menor resistência vascular e maior fluxo diastólico no território mesentérico do que recém-nascidos a termo. Tal diferença pode ser explicada pela baixa resistência vascular geral do prematuro ou pela baixa resistência vascular seletiva, devido ao seu rápido crescimento intestinal $^{(2)}$.

A vasodilatação funcional após a alimentação enteral em recém-nascidos de termo já é bem conhecida. Entretanto, o prematuro apresenta resposta variável, necessitando de compensações hemodinâmicas sistêmicas, como o aumento do débito cardíaco e a redução da pressão $\operatorname{arterial}^{(2)}$.

\section{Estudo de territórios vasculares}

O estudo de territórios vasculares desde a vida intrauterina, caracterizando a irrigação de órgãos, pode ser realizado por métodos não invasivos de imagem, utilizando-se o ultrassom com Doppler colorido ${ }^{(14)}$. Os principais territórios estudados no período neonatal são: cerebral, aórtico, renal, celíaco e mesentérico ${ }^{(15)}$.

Alterações do fluxo mesentérico superior e das respostas desse fluxo a algumas condições específicas estão relacionadas à evolução do recém-nascido, particularmente no que diz respeito à progressão da alimentação e ao desenvolvimento de quadros de intolerância, até de enterocolite necrosante ${ }^{(16)}$.

O fluxo sanguíneo mesentérico apresenta algumas respostas já conhecidas frente a situações diversas após o nascimento, tais como: vasodilatação depois da dieta, diferentes respostas perante a administração de drogas vasoativas, sepse e fototerapia. Tais respostas já são conhecidas em nascidos a termo e, no prematuro, espera-se resposta semelhante, porém não são conhecidas as características dessa resposta em diferentes idades gestacionais ${ }^{(17)}$.

\section{Mecanismos reguladores da circulação intestinal em recém-nascidos}

As características fisiológicas da circulação intestinal no recém-nascido são peculiares em relação às crianças maiores e adultos, havendo ainda distinção entre nascidos a termo e pré-termo. Essa circulação passa por mudanças dramáticas na evolução do estágio fetal para a condição neonatal e durante 
o primeiro mês de vida. Além disso, as respostas a variações das condições hemodinâmicas sistêmicas são também distintas às do adulto. Tais mudanças visam, principalmente, a preparar o intestino para assumir o papel da placenta na vida extrauterina, no sentido de captar nutrientes ${ }^{(16,17)}$.

O tônus basal vascular intestinal no recém-nascido é mais baixo, com resistência vascular menor, o que facilita um maior fluxo sanguíneo local. O aumento do fluxo sanguíneo intestinal no recém-nascido permite que o intestino mude da condição de um órgão dormente na vida intrauterina para um órgão de intensa atividade de crescimento, desenvolvimento e maturação( ${ }^{(2)}$.

Os mecanismos envolvidos na regulação do tônus basal do recém-nascido são distintos dos adultos. Classicamente, a regulação do tônus basal, assim como da resposta vascular em adultos, ocorre pelo sistema nervoso autônomo, principalmente por estimulação adrenérgica. Com base em estudos experimentais em suínos, duas fases da regulação são descritas em animais a termo saudáveis. Na primeira fase (até o primeiro mês de vida), a resistência vascular basal cai logo após o nascimento, atingindo um patamar estável e entrando, em seguida, na segunda fase (até os seis meses de vida), quando há elevação gradativa da resistência vascular até o início da adolescência, época em que atinge níveis semelhantes à do adulto ${ }^{(18)}$.

A regulação do tônus basal vascular no período neonatal precoce é determinada por um equilíbrio entre fatores constritores e dilatadores que atuam na musculatura lisa dos vasos intestinais. São caracterizados três sistemas de regulação: óxido nítrico (NO) constitutivo, que causa vasodilatação, resposta miogênica vascular, e endotelina-1 que causa vasoconstrição. Nessa fase, o tônus vascular basal e as alterações na resposta vascular não sofrem influência da estimulação adrenérgica extrínseca ${ }^{(19)}$.

A regulação vascular pelo NO constitutivo ocorre a partir da produção específica de NO pelas células endoteliais mediada por ação enzimática, seguida da difusão facilitada do NO para a musculatura lisa vascular, ativando a produção de GMP cíclico pela guanilato-ciclase, com redução do cálcio intracelular da musculatura lisa vascular e inativação do mecanismo contrátil. A produção de $\mathrm{NO}$ constitutivo local é contínua, podendo ser estimulada ou bloqueada química ou mecanicamente. Além disso, a velocidade de produção de NO depende da idade pós-natal, sendo acelerada do $3^{\circ}$ ao $12^{\circ}$ dia de vida ${ }^{(20)}$. A resposta de relaxamento vascular mediada por vasodilatadores NO-dependentes (acetilcolina, bradicinina e substância $\mathrm{P}$ ) é também mais acentuada nessa fase. A dilatação induzida pelo fluxo é um mecanismo indireto da ação do $\mathrm{NO}$ na vasodilatação mesentérica: o efeito mecânico do fluxo sobre a superfície celular endotelial estimula a ação enzimática ${ }^{(21)}$. O efeito da vasodilatação mesentérica mediada pelo NO diminui ao final do primeiro mês de vida.

A resposta miogênica vascular é um mecanismo no qual o aumento da pressão intravascular induz à vasoconstrição, representando uma resposta intrínseca da musculatura lisa vascular reflexa ao estiramento de suas fibras, e decorre, portanto, de estímulo mecânico. O mecanismo dessa resposta parece envolver a proteína $\mathrm{C}$ quinase (PKC) intracelular, com o aumento do influxo de cálcio para as células e contração das miofibrilas. A resposta miogênica é mais acentuada logo após o nascimento e cai gradativamente no final do primeiro mês de vida ${ }^{(21)}$.

A endotelina é um peptídeo vasoativo e mitogênico produzido principalmente pelo endotélio vascular. A sua ação ocorre por duas vias: ligação em receptores de endotelina A $\left(\mathrm{ET}_{\mathrm{A}}\right)$ da musculatura lisa vascular, o que leva à vasoconstrição potente, ou pela ligação em receptores de endotelina B $\left(\mathrm{ET}_{\mathrm{B}}\right)$, com vasodilatação mediada por NO. A endotelina-1 apresenta altas concentrações no período neonatal. Em condições basais, a ação mediada pelos receptores $\mathrm{ET}_{\mathrm{A}}$ é mais acentuada que pelos $\mathrm{ET}_{\mathrm{B}}$, permitindo a manutenção do tônus basal ${ }^{(20)}$.

Ao final do primeiro mês de vida, começam a declinar os efeitos da regulação do tônus vascular mediados por NO, resposta miogênica vascular e endotelina. Também nesse período, o tamanho, o peso e a superfície intestinal apresentam um ganho significativo, havendo grande proliferação e diferenciação vascular. Dessa forma, há aumento da resistência vascular devido à alteração geométrica dos vasos com aumento das ramificações. Além disso, a maturação do estímulo e da resposta a tal estímulo da inervação adrenérgica extrínseca é também responsável pelo aumento do tônus vascular ${ }^{(20)}$.

\section{Resposta vascular da circulação intestinal no recém-nascido}

Em decorrência das peculiaridades da resistência vascular basal e, consequentemente, do fluxo sanguíneo, esperam-se características específicas na resposta vascular frente à estimulação e a situações de estresse. Assim, respostas reflexas eficazes, por quimio e barorreceptores, são esperadas no intestino a partir de 30 dias de vida, devido à inervação adrenérgica extrínseca ${ }^{(21)}$. 
A inervação adrenérgica já está presente no nascimento, porém o seu amadurecimento ocorre a partir do final do primeiro mês de vida. Um estímulo adrenérgico no período neonatal causa uma vasoconstrição intestinal tênue e os vasos retornam ao tônus basal em até dois minutos após a estimulação - esse fenômeno é denominado escape autorregulatório e tem por objetivo preservar a irrigação local ${ }^{(22)}$.

Uma vez que o fluxo sanguíneo $(\mathrm{Q})$ em um órgão alvo é diretamente proporcional à pressão de perfusão $(\mathrm{P})$ e inversamente proporcional à resistência vascular $(\mathrm{R})$, segundo a fórmula $(\mathrm{Q}=\mathrm{P} / \mathrm{R})$, uma redução de pressão de perfusão até um nível fisiológico resulta em vasodilatação para manter a velocidade de fluxo constante, situação denominada autorregulação pressão-fluxo. Esse fenômeno não é observado nos primeiros dias de vida, estabelecendo-se apenas no final do primeiro mês. Como consequência, o intestino do recémnascido nos primeiros dias apresenta resposta ineficiente para manter o fluxo sanguíneo adequado frente a situações de hipotensão, com redução do aporte de oxigênio ${ }^{(20)}$. Esse efeito pode ser explicado pela baixa resistência vascular mesentérica nos primeiros dias de vida, fazendo com que a reserva frente a uma vasodilatação seja menor.

A resposta vascular intestinal diante da hipoxemia é variável e depende da taxa de oxigênio. Em situação de hipoxemia leve ou moderada, ocorre vasodilatação; já na hipoxemia grave, há vasoconstrição, o que agrava a isquemia tecidual. Essa resposta se deve, provavelmente, ao fato de que o oxigênio é um substrato essencial na produção do $\mathrm{NO}^{(22,23)}$.

A hiperemia pós-prandial caracteriza a resposta vasodilatadora do fluxo sanguíneo intestinal, com consequente aumento na liberação do oxigênio local em face da presença de nutrientes no lúmen. Nesse fenômeno, ocorre um aumento no número de capilares perfundidos, facilitando a difusão de oxigênio aos tecidos. Tal resposta é gerada por um mecanismo multifatorial, mas a principal participação é do sistema nervoso entérico, sendo a substância $\mathrm{P}$ o principal neurotransmissor envolvido ${ }^{(24)}$.

\section{Características da circulação intestinal na patogênese da enterocolite necrosante}

A enterocolite necrosante está presente em 2 a $7 \%$ das internações em Unidades de Terapia Intensiva Neonatal $(\text { UTIN })^{(25)}$. É uma doença multifatorial e, quanto mais prematura a criança, maior o risco. É também chamada de doença dos sobreviventes, pois geralmente ocorre quando o recém-nascido já superou as condições clínicas que o afligiram na fase aguda, logo após o nascimento, e inicia-se a alimentação enteral. Dentre os fatores de risco, destacamse: alterações de fluxo sanguíneo local e sistêmico (sepse, cateteres umbilicais, canal arterial patente, etc. ${ }^{(6,23)}$, lesão de reperfusão, presença de radicais livres, déficit enzimático, por exemplo, da enzima degradadora do fator antiplaquetário $(\mathrm{PAF})^{(8,9)}$, infecções, alimentação precoce, tipo e progressão inadequada da dieta ${ }^{(25,26)}$, infusão de drogas vasoativas ${ }^{(10,11)}$, entre outros. A taxa de mortalidade é de 20 a $40 \%$ e a frequência de complicações e sequelas, de 10 a $30 \%{ }^{(12,18)}$.

$\mathrm{Na}$ histologia, observa-se a presença de necrose de coagulação, particularmente nas camadas musculares intestinais, o que caracteriza um evento final do processo. A identificação do momento e do mecanismo de isquemia é importante para que se possa atuar na prevenção e no controle da ocorrência da enterocolite necrosante ${ }^{(27)}$.

A primeira teoria sobre o mecanismo de isquemia intestinal que leva ao desenvolvimento da enterocolite foi apontada por Lloyd em $1969^{(28)}$, que descreve a existência de perfuração intestinal em recém-nascidos com baixos escores de Apgar, o que sugeria, na época, asfixia ao nascimento. Essa teoria é justificada pela redistribuição de fluxo sanguíneo sistêmico com o objetivo de preservar órgãos nobres em situações de asfixia, levando à isquemia mesentérica. Estudos experimentais confirmaram essa teoria e, mais tarde, com base em observações clínicas, a mesma teoria foi criticada, uma vez que o principal fator predisponente da enterocolite seria a prematuridade, porque a doença geralmente ocorre após a primeira semana de vida, quando os efeitos da asfixia seriam menos proeminentes ${ }^{(29)}$. Além disso, com base nos estudos dos mecanismos envolvidos na fisiologia da circulação intestinal neonatal, é de se esperar pequeno efeito da asfixia na gênese da isquemia esplâncnica, pois o recém-nascido tem uma resposta adrenérgica e mecanismos autorregulatórios de escape para regular seu fluxo sanguíneo intestinal, como exposto anteriormente. Ademais, sabe-se que o dano tecidual decorre da reperfusão pós-isquemia ${ }^{(30)}$.

Atualmente, o mecanismo de isquemia mesentérica mais aceito se refere à lesão endotelial, uma vez que o controle do tônus e da resposta vascular decorre de fatores essencialmente citogênicos, sendo que um dos mais envolvidos é o NO. A redução do aporte de oxigênio leva à diminuição da quantidade de NO endógeno, enquanto a redução do fluxo sanguíneo diminui a resposta de produção estimulada do óxido nítrico. O NO interfere no receptor transmembrana da proteína Gligante do endotélio vascular, comprometendo a capacidade da ligação $\mathrm{K}$ e de inúmeros agonistas vasoconstritores, tais 
como norepinefrina, angiotensina II e endotelina-1, que são acoplados à ação da fosfolipase $C$. A ativação da fosfolipase $C$ é um passo essencial na gênese de mensageiros secundários que promovem a vasoconstrição ${ }^{(31,32)}$. A diminuição do NO reduz a ação vasodilatadora e libera os efeitos da vasoconstrição por ação mediada pela fosfolipase C. Os efeitos decorrentes da isquemia são observados no período neonatal precoce, não estando presentes após o final do primeiro mês de vida(33).

Outro mecanismo de vasoconstrição decorrente da lesão endotelial é a redução dos receptores $\mathrm{ET}_{\mathrm{B}}$, que teriam uma ação acoplada aos receptores de NO, aumentando a con-

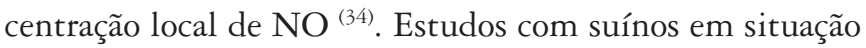
de isquemia leve e transitória indicam que uma redução de $10 \%$ da tensão de oxigênio sanguíneo seria suficiente para causar lesão endotelial, levando à vasoconstrição. No entanto, não haveria dano direto ao epitélio intestinal. Neste modelo, observou-se a redução de NO endógeno e a isquemia foi notada no terceiro dia de vida, não estando presente ao final do primeiro mês de idade ${ }^{(35)}$. Entretanto, quando ocorre isquemia mais profunda e prolongada, com redução de $50 \%$ da tensão de oxigênio por seis horas, além da redução do NO, nota-se um aumento da produção local de endotelina-1 que, aliada à redução dos receptores $\mathrm{ET}_{\mathrm{B}}$, exerce maior ação vasoconstritora ${ }^{(35)}$. A existência de maior concentração de ET-1 pós-isquemia mantém a situação de resistência vascular aumentada prolongada, mesmo após a reversão da isquemia ${ }^{(34)}$.

Há outros fatores que contribuem para o processo de isquemia na lesão endotelial: a presença do fator de ativação plaquetário, um lipídio mediador da inflamação ${ }^{\text {(36-38) }}$, a ocorrência da translocação bacteriana com liberação de toxinas próprias ou inflamatórias, a própria diminuição do trânsito intestinal com fermentação de açúcares no lúmen intestinal e a produção de ácidos graxos de cadeia curta, causando destruição da mucosa intestinal e propiciando o contato direto da microvasculatura com substâncias tóxicas ${ }^{(18)}$.

As hipóteses acima descritas se sustentam como mecanismos patogênicos da enterocolite necrosante baseados em constatações clínicas e histopatológicas: em fases iniciais da doença, observam-se ilhotas de isquemia e necrose na mucosa intestinal, antes de haver lesão extensa em toda a parede e em toda a extensão do intestino, o que seria esperado em caso de obstrução ou interrupção de fluxo sanguíneo. Além disso, modelos experimentais com indução de isquemia, hipofluxo e aumento ou redução de atividades enzimáticas e/ou inflamatórias produzem lesões intestinais semelhantes à enterocolite.

\section{Considerações finais}

A circulação mesentérica no período neonatal tem peculiaridades em relação à regulação de fluxo, ao tônus muscular, à resposta vascular fisiológica e a agressões. Tais peculiaridades devem ser levadas em conta na prática diária em relação ao jejum, ao tipo de alimentação e à progressão da dieta. Em relação às doenças clínicas gastrintestinais, a enterocolite necrosante é, sem dúvida, a mais prevalente, existindo correlação direta entre as peculiaridades da circulação mesentérica e a incidência e magnitude da enterocolite. A dopplerfluxometria da artéria mesentérica superior desponta como uma ferramenta útil para avaliar o território vascular e pode ser um parâmetro de indicação para a introdução e progressão da dieta em situações de risco para enterocolite necrosante.

\section{Referências bibliográficas}

1. Berseth CL. Developmental anatomy and physiology of the gastrointestinal tract. In: Taeusch HW, Ballard RA, editors. Diseases of the newborn. $7^{\text {th }}$ ed. Philadelphia: WB Saunders; 1998. p. 893-904.

2. Robel-Tillig E, Knüpfer M, Pulzer F, Vogtmann C. Blood flow parameters of the superior mesenteric artery as an early predictor of intestinal dysmotility in preterm infants. Pediatr Radiol 2004;34:958-62.

3. Nowicki PT. Ischemia and necrotizing enterocolitis: where, when, and how. Semin Pediatr Surg 2005;14:152-8.

4. Nowicki PT, Nankervis CA. The role of the circulation in the pathogenesis of necrotizing enterocolitis. Clin Perinatol 1994;21:219-34.

5. Crissinger KD. Regulation of hemodynamics and oxygenation in developing intestine: insight into the pathogenesis of necrotizing enterocolitis. Acta Paediatr Suppl 1994;396:8-10.
6. Kempley ST, Murdoch E. Splanchnic haemodynamic disturbances in perinatal sepsis. Arch Dis Child Fetal Neonatal Ed 2000;83:F139-42.

7. Havranek T, Madramootoo C, Carver JD. Nasal continuous positive airway pressure affects pre- and postprandial intestinal blood flow velocity in preterm infants. J Perinatol 2007;27:704-8.

8. Ewer AK. Role of platelet-activating factor in the pathophysiology of necrotizing enterocolitis. Acta Paediatr Suppl 2002;91:2-5.

9. Amer MD, Hedlund E, Rochester J, Caplan MS. Platelet-activating factor concentration in the stool of human newborns: effects of enteral feeding and neonatal necrotizing enterocolitis. Biol Neonate 2004;85:159-66.

10. Seri I, Abbasi S, Wood DC, Gerdes JS. Regional hemodynamic effects of dopamine in the indomethacin-treated preterm infant. J Perinatol 2002;22:300-5. 
11. Hoecker C, Nelle M, Poeschl J, Beedgen B, Linderkamp O. Caffeine impairs cerebral and intestinal blood flow velocity in preterm infants. Pediatrics 2002;109:784-7.

12. Hsueh W, Caplan MS, Qu XW, Tan XD, De Plaen IG, Gonzalez-Crussi F. Neonatal necrotizing enterocolitis: clinical considerations and pathogenetic concepts. Pediatr Dev Pathol 2003;6:6-23.

13. Pezzati M, Biagiotti R, Vangi V, Lombardi E, Wiechmann L, Rubaltelli FF. Changes in mesenteric blood flow response to feeding: conventional versus fiber-optic phototherapy. Pediatrics 2000;105:350-3.

14. De Cobelli F, Cappio S, Del Maschio A. Noninvasive imaging of the abdominal vessels. Rays 2001;26:269-76.

15. Kluckow M, Evans N. Superior vena cava flow in newborn infants: a novel marker of systemic blood flow. Arch Dis Child Fetal Neonatal Ed 2000;82:F182-7.

16. Pezzati M, Dani C, Tronchin M, Filippi L, Rossi S, Rubaltelli FF. Prediction of early tolerance to enteral feeding by measurement of superior mesenteric artery blood flow velocity: appropriate-versus-small-for-gestational-age preterm infants. Acta Paediatr 2004;93:797-802.

17. Fang S, Kempley ST, Gamsu HR. Prediction of early tolerance to enteral feeding in preterm infants by measurement of superior mesenteric artery blood flow velocity. Arch Dis Child Fetal Neonatal Ed 2001;85:F42-5.

18. Reber KM, Nankervis CA, Nowicki PT. Newborn intestinal circulation. Physiology and pathophysiology. Clin Perinatol 2002;29:23-39.

19. Reber KM, Mager GM, Miller CE, Nowicki PT. Relationship between flow rate and NO production in postnatal mesenteric arteries. Am J Physiol Gastrointest Liver Physiol 2001;280:G43-50.

20. Nankervis CA, Nowicki PT. Role of endothelin-1 in regulation of the postnatal intestinal circulation. Am J Physiol Gastrointest Liver Physiol 2000;278: G367-75

21. Nankervis CA, Dunaway DJ, Nowicki PT. Determinants of terminal mesenteric artery resistance during the first postnatal month. Am J Physiol Gastrointest Liver Physiol 2001;280:G678-86.

22. Robel-Tillig E, Vogtmann C, Bennek J. Prenatal hemodynamic disturbancespathophysiological background of intestinal motility disturbances in small for gestational age infants. Eur J Pediatr Surg 2002;12:175-9.

23. Bhatt AB, Tank PD, Barmade KB, Damania KR. Abnormal Doppler flow velocimetry in the growth restricted foetus as a predictor for necrotizing enterocolitis. J Postgrad Med 2002;48:182-5.
24. Nowicki PT. Postnatal changes in gut hemodynamics: a possible role for substance P. Am J Physiol 1998;274:G1142-50.

25. Reber KM, Nankervis CA. Necrotizing enterocolitis: preventative strategies Clin Perinatol 2004;31:157-67.

26. Carver JD, Saste M, Sosa R, Zaritt J, Kuchan M, Barness LA. The effects of dietary nucleotides on intestinal blood flow in preterm infants. Pediatr Res 2002;52:425-9.

27. Nowicki PT, Miller CE. Flow-induced dilation in newborn intestine. Pediatr Res 1995;38:783-91.

28. Lloyd JR. The etiology of gastrointestinal perforations in the newborn. J Pediatr Surg 1969;4:77-84.

29. Kliegman RM, Fanaroff AA. Necrotizing enterocolitis. N Engl J Med 1984;310:1093-103.

30. Nowicki PT. The effects of ischemia-reperfusion on endothelial cell function in postnatal intestine. Pediatr Res 1996;39:267-74.

31. Davis MJ, Hill MA. Signaling mechanisms underlying the vascular myogenic response. Physiol Rev 1999;79:387-423.

32. Parekh N, Dobrowolski L, Zou AP, Steinhausen M. Nitric oxide modulates angiotensin II and norepinephrine-dependent vasoconstriction in rat kidney. Am J Physiol 1996;270:R630-5.

33. Nowicki PT. Effects of sustained low-flow perfusion on the response to vasoconstrictor agents in postnatal intestine. Am J Physiol 1999;276:G1408-16.

34. Nankervis CA, Dunaway DJ, Miller CE. Endothelin ET(A) and ET(B) receptors in postnatal intestine. Am J Physiol Gastrointest Liver Physiol 2001;280: G555-62.

35. Nowicki PT, Miller CE, Hayes JR. Effect of sustained mesenteric nerve stimulation on intestinal oxygenation in developing swine. Am J Physiol 1991;260:G333-9.

36. Rand T, Weninger M, Kohlhauser C, Bischof S, Heinz-Peer G, Trattnig S et al. Effects of umbilical arterial catheterization on mesenteric hemodynamics. Pediatr Radiol 1996;26:435-8.

37. Weir FJ, Ohlsson A, Fong K, Amankwah K, Coceani F. Does endothelin-1 reduce superior mesenteric artery blood flow velocity in preterm neonates? Arch Dis Child Fetal Neonatal Ed 1999;80:F123-7.

38. Caplan MS, Sun XM, Hseuh W, Hageman JR. Role of platelet activating factor and tumor necrosis factor-alpha in neonatal necrotizing enterocolitis. J Pediatr 1990;116:960-4. 